\title{
Epidemiology based study on the risk factors of breast cancer research: the Bangladesh perspective
}

\section{Opinion}

Breast cancer starts in the cells of the breast and grows aberrantly. Most often, breast cancer begins in the tissue of the milk ducts or in the lobules that supply milk to the ducts. It is reported as the most recognized cancer in woman and the second highest in terms of mortality rates worldwide. ${ }^{1}$ Recent global cancer statistics states that breast cancer incidence is growing at a faster rate in developing countries. ${ }^{2}$ The early diagnosis and detection method of breast cancer treatment is crucial in Bangladesh. It may be possible by making people beware of the risk factors associated with breast cancer and its proactive effect through conducting various epidemiology based public health studies in different regions of Bangladesh. The early identification of cancer increases the chances of successful treatment to a greater extent.

\section{Breast cancer in Bangladesh perspectives: Recent scenario}

In 2016, an epidemiology study ${ }^{3}$ was conducted on breast cancer among the female patients in Bangladesh. The study indicated that $90 \%$ respondents practice breastfeeding their baby. Around $56 \%$ respondents had the parenchymal pattern of the breast in mammography. The study also revealed that less than $50 \%$ respondents had the history of disease on breast cancer, $25 \%$ had the history of benign breast cancer and $2 \%$ had the experiences to use radiation. Another study ${ }^{4}$ showed that knowledge about breast cancer screening was seemingly high, but only a few respondents were conversant with the diagnosis methods of breast cancer. Only 5 respondents had the idea about the required age and frequency for performing mammography. The study also reported that the minority of respondents (about 38\%) knew the crucial role of nutritious food in lowering the risk. A group of researchers performed a hospital based epidemiology study at National Institute of Cancer Research and Hospital (NICRH) in Dhaka and identified physical inactivity, being menopause, positive family history of breast cancer, and history of induced abortion as significant risk factors. ${ }^{5}$ The study recommended longer duration of breastfeeding and special attention for breast cancer prevention.

\section{Summary}

Despite suffering from breast cancer, most of the women in Bangladesh diagnose it at late stages, especially in metastasis condition when the cure is impossible. Taking the current scenario into account, the following initiatives may be handy to prevent and minimize the breast cancer disease in Bangladesh:

a. Diagnosis at the early stage before the condition deteriorates and taking treatment as prescribed by the physician

b. Developing risk prediction software to visualize risk factor score early using data mining technology

\author{
Volume 7 Issue 5 - 2018
}

\author{
Md Shariful Islam,' Md Mohsan Khudri² \\ 'Department of Biotechnology and Genetic Engineering, \\ Mawlana Bhashani Science and Technology University, \\ Bangladesh \\ ${ }^{2}$ Department of Business Administration, Uttara University, \\ Bangladesh
}

Correspondence: Md. Mohsan Khudri, Department of Business Administration, Uttara University, Uttara, Dhaka-I230, Bangladesh, Email mkhudri@isrt.ac.bd

Received: June 04, 2018| Published: September 06, 2018

c. Raising the consciousness of people through various community campaigns

d. Preparing nationwide cancer registry report annually

e. Carrying out region-wise epidemiology based study on a regular basis

\section{Acknowledgements}

Author declares that their no conflict of interest.

\section{Conflict of interest statement}

The author declares that this Opinion was written in the absence of any commercial or financial relationships that could be construed as a potential conflict of interest.

\section{References}

1. Chander A, Qureshi NA, Sodhi BS, et al. Epidemiology of Breast CancerA Hospital Based Study. Int J Sci Stud. 2017;5(6):63-66.

2. Bray F, McCarron P, Parkin DM. The changing global patterns of female breast cancer incidence and mortality. Breast cancer Res. 2004;6(6):229 239.

3. Bellah SF, Salam MA, Karim MR, et al. Epidemiology of breast cancer among the female patients in Bangladesh. Orient Pharm Exp Med. 2016;16(2):85-95.

4. Nizum NA. A Study on Breast Cancer Awareness and Knowledge among Rural Female Population of Bangladesh. A dissertation submitted to the Department of Pharmacy, East West University, Bangladesh. 2014;1-89.

5. Jabeen S, Haque M, Islam M, et al. Breast cancer and some epidemiological factors: a hospital based study. J Dhaka Med Coll. 2013;22(1):61-66. 